\title{
artículos
}

\section{El problema de las transformaciones de cines y tea- tros en España. La metamorfosis de un edificio emble- mático: de Teatro Kursaal a Cine Nacional}

Antonio Bravo Nieto

Universidad de Málaga

PALABRAS CLAVE: Arquitectura s. XX/ Edificios para teatros/ Melilla

\section{RESUMEN}

La construcción de grandes y monumentales edificios destinados a cines y teatros, fue una de las más sugerentes aportaciones arquitectónicas de la primera mitad del siglo XX. El teatro-cine Kursaal de Melilla es un ejemplo de cómo estas construcciones asumieron un importante papel de modernidad y fueron reflejo de las últimas y más atrevidas tendencias estéticas de su época. El Kursaal fue construido con proyecto del arquitecto Enrique Nieto a partir de 1929, y en sus formas encontramos el último canto del cisne del modernismo más geométrico y desornamentado, preludio de la arquitectura art déco. Su evolución a lo largo del siglo XX, también ilustra sobre la variable suerte y conservación de estos edificios en tiempos tan cambiantes y su presente y futuro es un reto sobre la necesidad de conservar el patrimonio cultural de las ciudades.

\section{ABSTRACT}

The construction of large and monumental buildings planned for cinemas and theatres was one of the most evocative architectural contributions in the first half of XXth century. The theatre-cinema Kursaal in Melilla is an example of how these buildings have assumed the key role of modernity and were reflection of the latest and daringaesthetic tends of that time. The Kursaal was built as a project of the architect Enrique Nieto from 1929 and in its shapes we find the latest swan song of of the most geometric and bare of ornament Modernism, prelude to art deco architecture. Its evolution during the XXth century, also illustrates about the variable luck and conservation of these buildings in such changing times and its present and future is a challenge on the need to preserve cities' cultural heritage

En la segunda mitad de los años veinte se produce en España un fenómeno muy generalizado y acorde con la importancia que algunas ciudades iban adquiriendo en su configuración urbana y arquitectónica, al amparo de la bonanza económica. En este panorama, asistimos a la construcción de grandes espacios para el ocio,

* BRAVO NIETO, A.: "El problema de las transformaciones de cines y teatros en España. La metamorfosis de un edificio emblemático: de Teatro Kursaal a Cine Nacional", Boletín de Arte, $\mathrm{n}^{\circ} 28$, Departamento de Historia del Arte, Universidad de Málaga, 2007, págs. 252. 
entre los que se encuentran cines y teatros que con su monumentalidad asumían la imagen de nuevos templos en el perfil construido de muchas ciudades. Por esta razón, todas las grandes capitales del país van a contar con proyectos señeros que constituyen una parte importante de su propia memoria colectiva.

Este es el caso evidente de la ciudad de Melilla, pues en los primeros decenios del siglo $\mathrm{XX}$ fue una urbe cosmopolita y bulliciosa en la que se materializaban las necesidades de una sociedad que demográficamente se mostraba muy pujante y exigía grandes espacios públicos ${ }^{1}$. Así, en estos momentos se construyen los tres grandes teatros-cines melillenses: el Perelló, con proyecto del ingeniero Luís GarcíaAlix (1927-1928), el Kursaal-Nacional obra del arquitecto Enrique Nieto Nieto (19291931) y el cine Monumental del también arquitecto Lorenzo Ros Costa (1930-1932)2.

\section{LA GÉNESIS DEL PROYECTO Y SU MATERIALIZACIÓN.}

El Kursaal fue un proyecto empresarial que ya arranca desde 1912, año en el que se levantaba en una de las manzanas del ensanche central de Melilla ${ }^{3}$ un pabellón de espectáculos erigido con materiales no permanentes. Este teatro provisional atraía al público melillense que deseaba contemplar los espectáculos de diferentes compañías de variedades y en el que también se realizaban algunas proyecciones cinematográficas. Desde 1922 el propietario del solar y empresario, Rafael Rico Albert, animado por el auge social que el cine iba adquiriendo, lo dedicó exclusivamente a sala de proyección y a finales de este decenio decidió renovar estas instalaciones de madera e invertir en la construcción de un edificio de gran monumentalidad. El arquitecto elegido para realizar el encargo fue el técnico barcelonés afincado en Melilla Enrique Nieto Nieto que ejecutó el proyecto de teatro ${ }^{4}$ y la dirección de las obras de su construcción.

1 Vid. el libro de DíEZ SÁNCHEZ, J. (1997). Melilla y el mundo de la imagen. Aproximación a la fotografía, el cine y la televisión. Melilla: Servicio de Publicaciones de la Ciudad Autónoma.

2 Sobre los teatros melillenses existe una bibliografía interesante: el Cine Monumental ha sido el más estudiado, y cuenta con algunas investigaciones concretas: PÉREZ ROJAS, F. J. (1987): "Sobre la arquitectura del cine en España. El cine Monumental Sport de Melilla". En: El barco como metáfora visual y vehículo de transmisión de formas. Actas del Simposio Nacional de Historia del Arte (CEHA). Málaga-Melilla, 1985. Málaga: varios; págs. 283-287. CAMACHO MARTíNEZ, R. (1986). "Las sugestiones del Art Déco en Melilla". Málaga: varios; págs. 283-287. CAMACHO MARTiNEZ, R. (1986). "Las sugestiones del Art Déco en Melilla".
Boletín de Arte, $n^{\circ} 7$; págs. 155-167. El caso del teatro Perelló y del cine teatro Kursaal han merecido hasta el momento menos atención: BRAVO NIETO, A. (1996). La construcción de una ciudad europea en el contexto norteafricano, arquitectos e ingenieros en la Melilla contemporánea. Málaga-Melilla: Universidad-Ciudad Autónoma.

3 DÍEZ SÁNCHEZ, J. (1997).Op.Cit. pág. 111 a 113.

4 Le agradecemos a D. José Ma Pérez Hurtado, que fue durante muchos años gerente del cine la amabilidad con la que nos ha mostrado diferentes documentos y planos del edificio, así como sus apreciaciones personales sobre el Nacional. 


\section{- E artículos El problema de las transformaciones de cines y teatros...}

1. Planta baja del proyecto, diciembre de 1929.

Enrique Nieto firma el proyecto en diciembre de 19295, cuando ya era un profesional totalmente consagrado en la ciudad, donde había construido numerosos e importantes edificios. En su propuesta podemos observar una serie de características que definen claramente el edificio en sus líneas decorativas fundamentales, marcadas por lo detalles ornamentales geométricos que generan una concepción monumentalista de la obra. El contratista de las obras fue Pedro Martínez Rosa, uno de los principales empresarios del sector y colaborador habitual de Nieto, actuando en la carpintería Adolfo Hernández y en la ornamentación Vicente Maeso, que también había colaborado ampliamente con el arquitecto Nieto en proyectos anteriores. Salvados los correspondientes permisos, en mayo de 1930 se iniciaba el derribo del antiguo salón provisional y comenzaban las obras del nuevo edificio. El Kursaal se inauguró el 14 de abril de 1931, situado en un lugar estratégico del ensanche, entre las calles Pablo Vallescá y la de Joaquín Costa (posteriormente Cándido Lobera) a la que tendría su fachada principal.

Respecto al estilo elegido, habría que realizar algunas apreciaciones que nos permitan entender sus formas y porqué fueron elegidas. Enrique Nieto había sido en Melilla el defensor a ultranza de un modernismo floralista muy en la línea de las obras de Lluis Domènech i Montaner, pero ya en los años veinte había variado sus

5 Sobre Enrique Nieto y la arquitectura de Melilla existe una amplia bibliografía. Véanse como estudios generales del periodo la obra de BRAVO NIETO, A. (1996): Op. Cit. que analiza el panorama arquitectónico general de la ciudad y la de GALLEGO ARANDA, S. (1996): Enrique Nieto en Melilla, la ciudad proyectada. Granada-Melilla: Universidad-UNED y del mismo autor (2005). Enrique Nieto (1880-1954) Biografía de un arquitecto. Melilla: Fundación Ciudad Monumental, ambas obras centradas en los aspectos biográficos del arquitecto. 


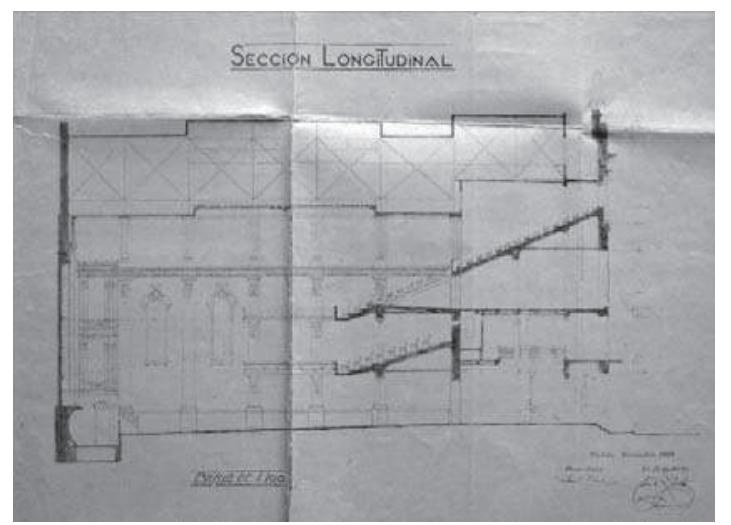

códigos estéticos y ensayaba nuevas formulaciones que, sin renunciar al modernismo, le alejaban de los postulados más floralistas de éste. Por estas fechas, se apreciaba la necesidad de abandonar esta línea, pero no existía ninguna condición para rechazar en sí mismo el concepto de una arquitectura ornamentada. Por esa razón, Nieto elige para la fachada principal (calle Cándido Lobera) un planteamiento sobrio, con un rotundo cuerpo central abierto con gran vano en arco segmentado y compartimentado (al gusto de la corriente modernista más centroeuropea), que servía de asiento a una rotunda hilera de pilares que soportan un entablamento con pequeño frontón ligeramente curvado y un potente remate con pináculos de gran esbeltez. Por su parte, una discreta balconada curva, daba el contrapunto horizontalista necesario en la composición. Los cuerpos simétricos laterales de esta fachada principal, mucho más discretos en su concepción, se asumen con un gran vano muy estilizado verticalmente entre potentes pilastras de orden gigante que rematan en pináculos romos. No faltan de todos modos algunos elementos florales muy contenidos, en la coronación de las pilastras o en los detalles de sobreventanas. La visión general de la fachada ofrece un impecable ritmo modernista en la ondulación de los vanos principales, pero sin romper el esquema geométrico y lineal del conjunto.

Estos modelos compositivos tienen sus raíces más directas en la arquitectura modernista centroeuropea, más concretamente en la Sezeción vienesa, que aporta el ideal de la geometrización ornamental al servicio de la elegancia. Sus formas fueron ampliamente difundidas por toda Europa y por España, donde encontramos su huella en diferentes arquitecturas ya desde el primer decenio del siglo XX. La fachada del Kursaal nos recuerda la composición de vano segmentado compartimentado por pilares que aparece en diferentes edificios de gran envergadura y en arqui- 
tecturas industriales. Por poner un ejemplo alejado de la realidad melillense, citaremos un proyecto del arquitecto Julio Galán para Cine Teatro en el parque de San Francisco de Oviedo, estudiado por la profesora María Cruz Morales Saro ${ }^{6}$. También existen en Melilla varias arquitecturas fabriles en el barrio Industrial que siguen el mismo esquema sezesionista, algunas ya desaparecidas en el barrio Industrial, conservándose los Almacenes Montes como el edificio más singular que sigue estos parámetros.

La fachada lateral del Kursaal, calle Pablo Vallescá, se despliega en un espacio mayor y aparece determinada por un ritmo verticalista formado mediante estrechos vanos muy alargados entre grandes pilastras que rematan por encima del entablamento en pináculos de coronación roma. A su vez, una moldura a modo de bocel recorre horizontalmente las dos fachadas abrazando los arcos superiores de los vanos y confiriendo un elemento de equilibrio compositivo en un conjunto que potencia sobre todo los elementos verticales.

Es obvio por tanto que el programa decorativo que presenta el edificio nos lleva hacia vertientes muy geométricas de la ornamentación modernista, que sin desterrar totalmente sus formas, sí que llegan a simplificar sus motivos, con vanos curvados y róleos simples.

Enrique Nieto no quiso o no pudo desplegar en esta obra un lenguaje Art Déco que ya se imponía en buena parte de Europa, y prefirió utilizar formas del modernismo centroeuropeo que se adaptaban al ideal de sobriedad y geometrización que empezaba a imponerse por entonces. La Sezeción, a finales de los años veinte podía entenderse como una tendencia general que si no encajaba totalmente dentro del Art Déco, sí podía considerarse como convergente con él. Nieto elabora el proyecto en un momento en el que no ha ensayado suficientemente la nueva tendencia decorativa, por lo que prescinde totalmente de sus motivos a la hora de plantear el programa decorativo de este edificio. Sin embargo, un año después, el arquitecto Lorenzo Ros va a realizar un extraordinario despliegue de arquitectura y motivos Art Déco en el Cine Monumental ${ }^{7}$, a escasos metros del anterior, demostrando que el modernismo, en cualquiera de sus vertientes, había sido desplazado por una nueva sensibilidad decorativa.

El estudio de la documentación y de los proyectos originales nos sitúa ante un edificio ejecutado en dos momentos diferentes, uno en el proyecto firmado en 1929 y el segundo que se realiza en 1934. En el primer proyecto Nieto diseñaba el cuerpo principal del edificio, con sus dos fachadas, la principal a Cándido Lobera y

6 MORALES SARO, M. C. (1989): El modernismo en Asturias. Arquitecturas, escultura y artes decorativas. Oviedo,Colegio Oficial de Arquitectos de Asturias, pág. 53.

7 CAMACHO MARTíNEZ, R. (1986) Op.cit. y PEREZ ROJAS, F. J. (1987) Op.Cit. 


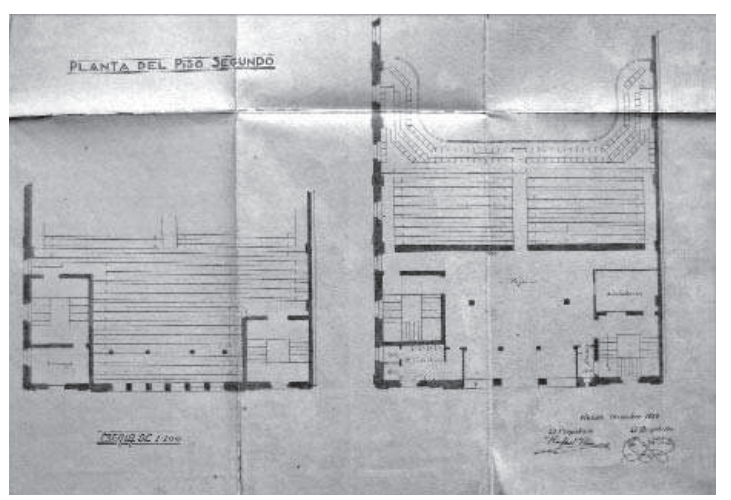

lateral a Pablo Vallescá, la parte de vestíbulo, patio de butacas, los dos pisos de palcos y parte del escenario, pero dejaba para un segundo momento los camerinos y trasera del escenario, que son construidos como edificio anexo al anterior, ofreciendo una fachada también con vista a Pablo Vallescá muy desornamentada y sobria que no sigue el ritmo del cuerpo principal.

La estructura constructiva del edifico se basa en elementos muy sólidos: muros maestros de gran capacidad portante, mixtos de ladrillo y mampostería, y estructura interior sustentada fundamentalmente en elementos metálicos: pilares, columnas y vigas de hierro son la parte fundamental del aparato portante. El análisis actual de la estructura, demuestra un estado de conservación muy aceptable.

No sabemos muy bien por qué razón el propietario, D. Rafael Rico Albert no planteó unitariamente el edificio y encargó dos proyectos consecutivos en el tiempo: diciembre de 1929 el primer proyecto correspondiente a la parte principal y más noble del edificio 8 y noviembre de $1934^{9}$ el segundo, correspondiente al solar trasero al teatro, donde irían situados todos los camerinos. El teatro fue inaugurado provisionalmente en abril de 1931 con la proyección de una película y en 1935 se inauguró la segunda parte de las obras, correspondientes al nuevo escenario, completándose en ese año la construcción en su estado definitivo. Diremos que estamos ante un mismo edificio concebido en dos momentos distintos, con dos proyectos diferentes, pero con una unidad cronológica en el desarrollo de las obras, fruto posiblemen-

8 Proyecto de Cine Kursaal. Diciembre de 1929. Arquitecto Enrique Nieto Nieto. Planos de ubicación, fachada, sección longitudinal, planta baja, planta del piso segundo y general. Archivo de D. José Ma Pérez Hurtado.

9 Proyecto de escenarios para el Cine Kursaal, noviembre de 1934. Arquitecto Enrique Nieto Nieto. Planos de fachada, sección transversal, sección longitudinal, planta del foso, planta del escenario. Archivo de D. José Ma Pérez Hurtado. 


\section{- E a tículos El problema de las transformaciones de cines y teatros...}

\section{El Kursaal en su} in a u guración,

Memoria de la Junta Municipal, 1931.

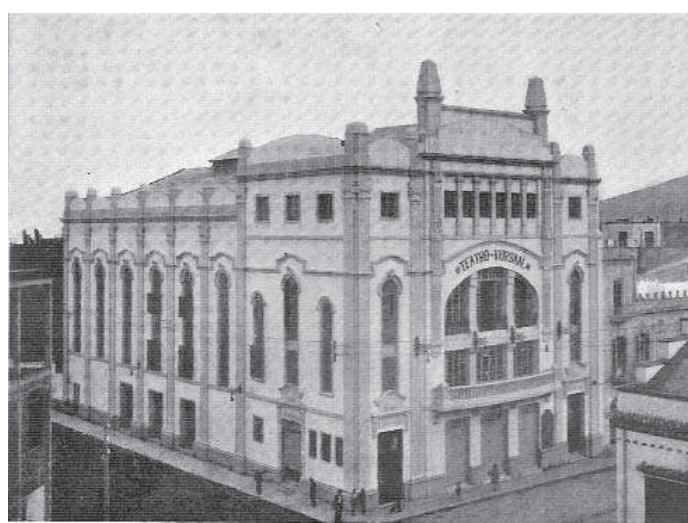

te de las expectativas comerciales del propietario que iría determinando el encargo al arquitecto.

Un aspecto interesante que conviene destacar es la dualidad que desde el principio del encargo va a producirse en la concepción del edificio: ¿teatro o cine? Esta dualidad, ha generado a lo largo de la historia del Kursaal ciertos problemas, reformas, actuaciones y modificaciones a los proyectos originales, debido a la incompatibilidad que a veces se genera entre ambos usos. Iremos desarrollando este problema a lo largo de estas notas, pero interesa plantearlo aquí como punto de partida, puesto que es una premisa importante a la hora de iniciar la recuperación actual del edificio.

El edificio original constaba de un gran patio de butacas en el bajo, una galería de butacas de Principal en el piso primero y otra en el segundo de General. Estos dos pisos disponían de palcos sobre ménsulas que asumían una planta de formas curvas en sus laterales, de elegante diseño. También contaba el teatro con dos palcos en proscenio, a cada lado del escenario, dispuestos en dos pisos y de planta curva, que permitiría una privilegiada asistencia a las representaciones teatrales. Estaban ricamente decorados si nos atenemos al proyecto original, con columnas o pilastras ornamentadas con cierta elegancia.

La decoración interior era simple pero destacaban algunos detalles en los palcos y sobre todo en los proscenios. Los muros laterales de la gran sala disponían de pilastras adosadas de orden gigante y remate con detalle modernista, y entre paños unos vanos simulados con remate curvo, muy similares a los dispuestos en el exterior, con paños decorativos de celosía.

Por otra parte, nos encontramos ante un edificio de gran envergadura, de 


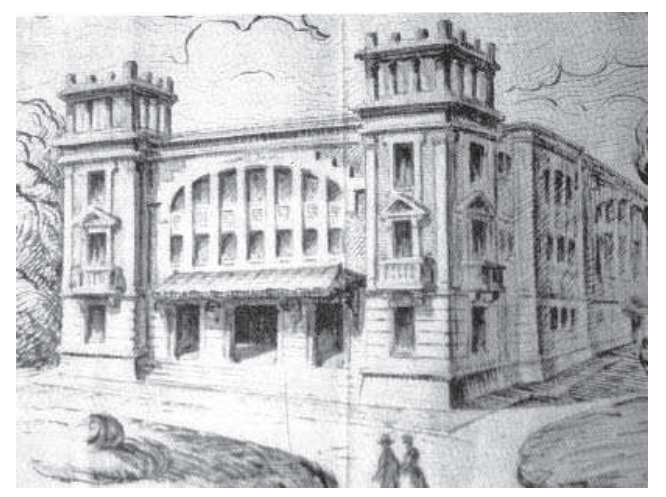

5. Proyecto de cine en Oviedo, publicado por la profesora $M^{a}$ Cruz Morales Saro, op. cit.

unos 23.000 metros cúbicos edificados y que en algunos momentos de su historia, sumando todas las butacas del patio, del principal y del general, hacían posible una capacidad de 2.000 localidades de aforo sentado. Sin duda una obra singular con vocación de monumentalidad.

\section{LAS TRANSFORMACIONES DEL EDIFICIO.}

El teatro Kursaal ha sufrido a lo largo de su historia algunas transformaciones que deben ser estudiadas, puesto que explican diversas circunstancias que no se pueden ignorar y que permiten hacer comprensible la forma en la que esta arquitectura nos ha llegado hasta nuestros días. La primera reforma que sufrió el edificio vino dada por el hecho de no contar en el proyecto original con la existencia de una cabina de proyección cinematográfica, lo que exigió posteriormente que se tuviera que construir esta cabina de forma superpuesta al proyecto original.

También sufrió transformaciones propias de los tiempos difíciles que determinaron la historia española de los años treinta y durante la guerra civil cambió el nombre de Kursaal pasando a llamarse Nacional, denominación más acorde con los nuevos aires triunfalistas que se respiraban por todo el país.

Durante los años treinta y cuarenta el edificio no sufre modificaciones notables y en una fotografía de 194410 tenemos una vista del interior antes de las agresivas transformaciones posteriores. Observamos en esta imagen, tomada desde el

10 Imagen en la revista Mundo Ilustrado, n 85-86, marzo 1944; s. p.: "Espectáculos de Melilla", aparece una foto del exterior "Edificio del gran Cine-Teatro Nacional, que por su magnificencia da ornato a la ciudad" y otra del interior: "La suntuosidad del gran salón de espectáculos del Cine-Teatro Nacional, queda bien manifiesta con esta foto". 

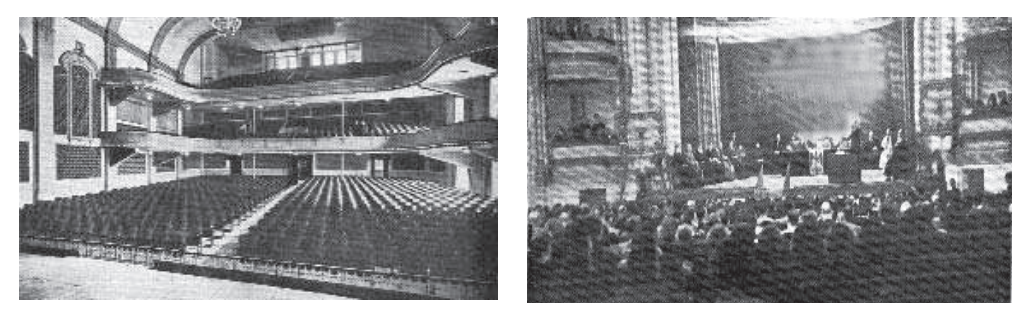

6. Vista interior con los dos pisos de gradas y palcos. Mundo Ilustrado 1944.

7. Conferencia de Feliciano Laverón, 1948. Se observan los palcos proscenios..

escenario, dos pisos de palcos: el principal y el general, con la planta curvada en los extremos más cercanos a los muros laterales y apoyados sobre ménsulas muy alargadas, así como ciertos detalles decorativos en el frontal de los palcos. Respectos a las paredes maestras, se mantenían como se proyectaran en 1929: grandes pilastras recorrían los muros hasta el nivel de cornisa, con las características líneas secesionistas en su parte superior. Desde la línea de cornisa arrancaba un falso techo como bóveda rebajada, con arcos fajones de escayola que volteaban desde las pilastras. Techo del que pendían varias lámparas.

A partir de los años cincuenta comienzan a producirse algunas transformaciones significativas ${ }^{11}$. El edificio, concebido fundamentalmente como teatro, ofrecía algunos problemas de visualidad que se intentó solucionar mediante varias obras posteriores. La imposición del cine frente al teatro empezaba a ser una evidencia por entonces. Aspectos que en 1929 no se tuvieron en cuenta empezaban ahora a ser prioritarios, como el tamaño de la pantalla para proyección de las películas, puesto que la embocadura original era excesivamente pequeña para sistemas panorámicos o de cinemascope. La evolución de la propia industria cinematográfica exigió reformas que son las que han dado lugar al edificio tal y como lo conocemos hoy día. De este periodo es interesante un proyecto que estudia precisamente el tamaño de la embocadura y pantalla, y su necesaria ampliación lo que finalmente conllevaría la supresión de importantes elementos del interior.

En 1952-195312 se realiza una importante transformación de la decoración interior, con la supresión de los detalles ornamentales realizados por Enrique Nieto en 1929. Así se "modernizaba" el cine, ganando en elegancia y en calidad de los

11 Proyectos y fotografías del Archivo de D. José Ma Pérez Hurtado.

12 Fotografías del Archivo de D. José Ma Pérez Hurtado. 


2: artículos Antonio Bravo Nieto
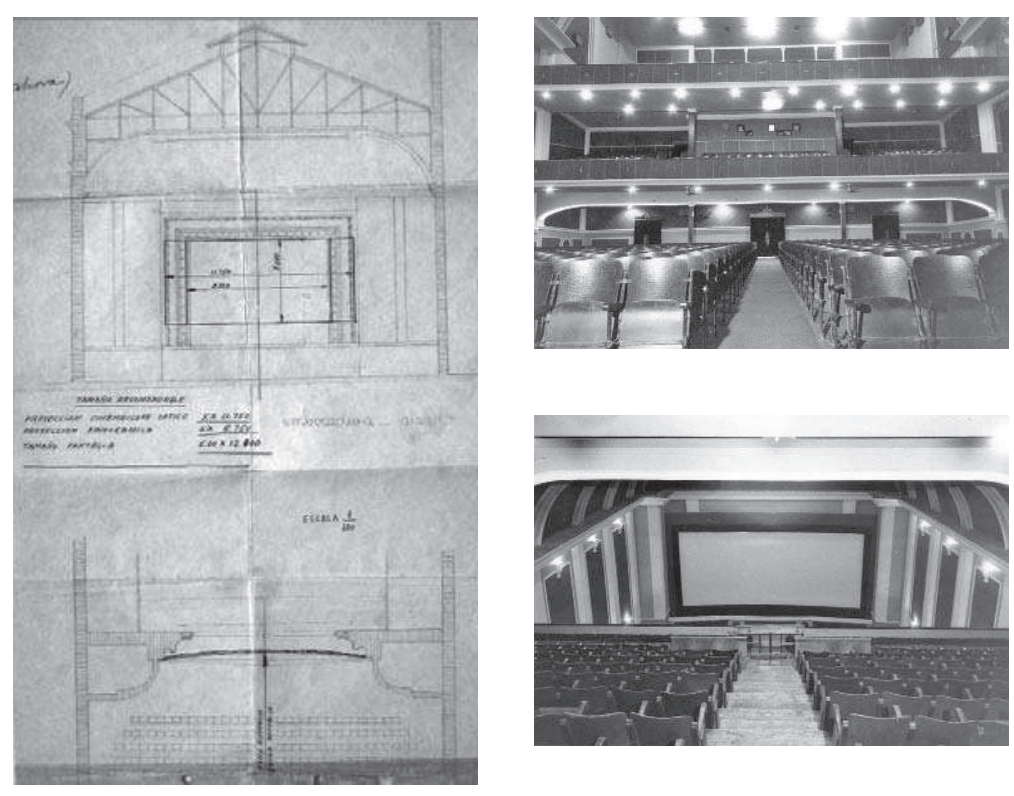

8. Proyecto de modificación de la embocadura de la pantalla. 1952-1953.

9. Vista de las gradas después de las obras de reforma, 1969.

10. Vista de la pantalla y escenario desde las gradas superiores, 1969.

nuevos materiales utilizados, pero a costa de los detalles ornamentales originales, desapareciendo la forma de las pilastras, la decoración de los vanos entre paños y los detalles de remate curvo. La obra representó una nueva forma de decoración con escayolas, luces, molduras y telas, realizada por un prestigioso decorador levantino llamado Aroca, que remodeló visualmente el interior del cine de acuerdo a los gustos imperantes en los años cincuenta. Es evidente que el edificio ganaba en comodidad, pero perdía una decoración original que contextualizaba el interior con el estilo del exterior.

Los palcos proscenios dejaron de tener sentido en un momento en el que el teatro ya no tenía la importancia del pasado y el cine se imponía como espectáculo de masas, por lo que se cegaron estos palcos junto al escenario, aunque durante 
unos años se mantuvo curiosamente su volumetría. A estos proscenios se accedía por escaleras independientes y a pesar de quedar obsoletos por la exigencia de crecimiento de la pantalla, daban un carácter muy especial al escenario. Todavía en fotos de 1969 podemos apreciar su espacio volumétrico en los laterales del escenario, ya convertido completamente en pantalla.

Entre 1958-1959 también se produjo otra reforma importante ${ }^{13}$. Momento en el que se sucedieron en la ciudad varios terremotos, la gerencia del cine decidió que los pináculos de la fachada principal podían resultar peligrosos al estar constituidos por materiales muy pesados y por ser su base frágil. Esto llevó a una obra en la que se demolieron estos remates verticalistas de fachada, lo que desfiguró bastante la obra original, restándole monumentalidad. Según datos aportados, el volumen de lo demolido consistió en varios camiones de escombros, lo que nos da idea de la obra efectuada.

En 1969 se produce la última gran reforma del edificio ${ }^{14}$. Nuevas necesidades del cine exigieron una nueva ampliación de pantalla y con la participación del mismo decorador, Aroca, se acometió la eliminación definitiva de los palcos proscenios de la embocadura, dando lugar a una gran pantalla. Reforma por entonces necesaria, pero muy agresiva con el diseño original que perdía irremediablemente estos palcos

También se acometió por entonces la reforma de los pisos superiores, eliminando los remates curvos de los palcos que se habían convertido en "obstáculos" que dificultaban la visualización de toda la pantalla por parte del público que se situaba en la zona de principal y general. Por esta razón se hizo recto el frente de estos palcos, amputando el desarrollo curvo de su planta.

Es totalmente cierto que el cine perdía entonces buena parte de la elegancia que los palcos curvos conferían al interior. Se imponía la aplastante lógica de las necesidades de modernización, pero también desde nuestra perspectiva se sacrificaba un espacio monumental original, determinando finalmente que el interior del edificio actual no se corresponda estilísticamente con el estilo modernista geométrico que presenta en sus fachadas externas, sino más bien a modelos propios de los años cincuenta o sesenta.

Una nueva etapa se abre para este edificio monumental cuando la industria del cine tradicional entra en una profunda crisis que está llevando a una de las mayores destrucciones o modificaciones del patrimonio que se están viviendo en la actualidad: las demoliciones o actuaciones de adaptación de estos teatros o cines en nuevos edificios de múltiples funciones. El futuro del cine Nacional parece estar asegu-

13 Proyectos y fotografías del Archivo de D. José Ma Pérez Hurtado.

14 Proyectos y fotografías del Archivo de D. José Ma Pérez Hurtado. 


2: artículos Antonio Bravo Nieto
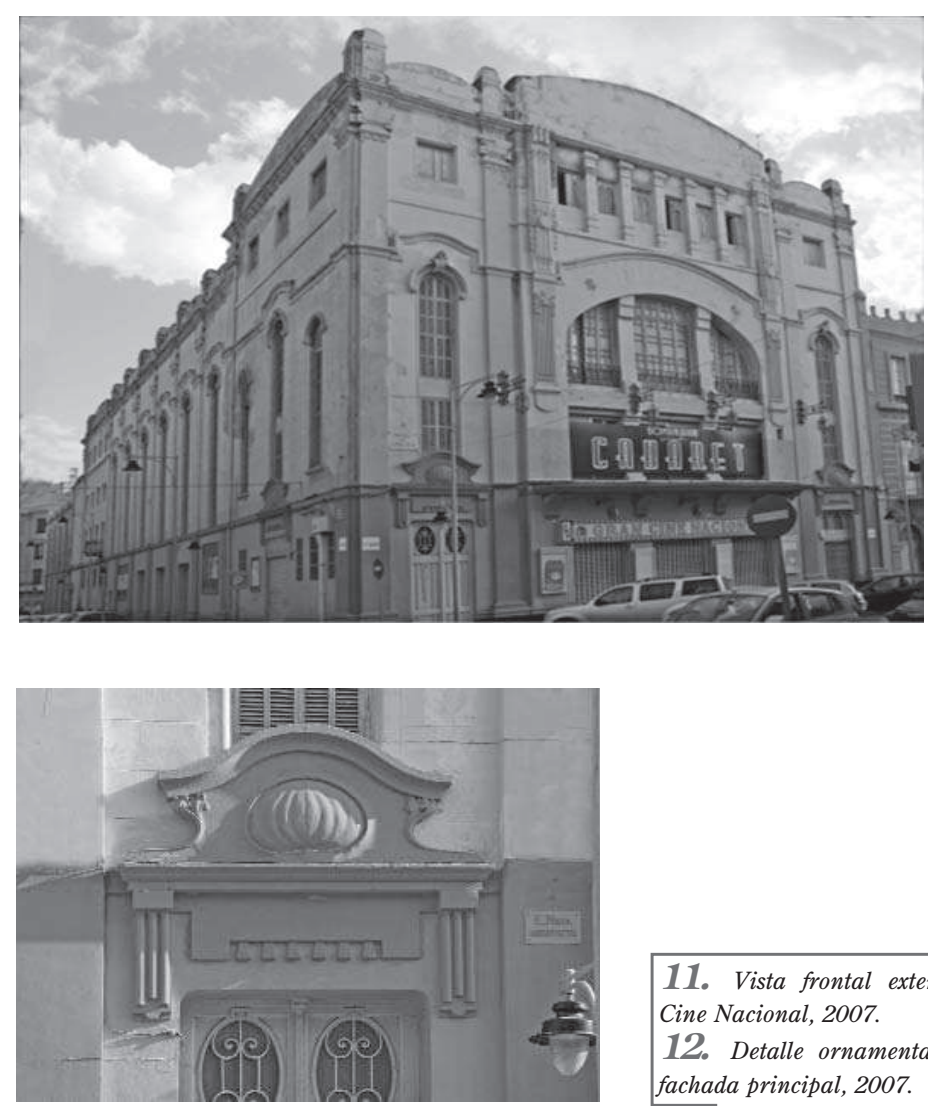

11. Vista frontal exterior del Cine Nacional, 2007.

12. Detalle ornamental de la fachada principal, 2007.

rado al haberse adquirido recientemente por parte de la Ciudad Autónoma de Melilla para ubicar el futuro teatro público de la ciudad, con lo que es de esperar que sus formas originales puedan ser conservadas y restauradas y vuelvan a resurgir con la importancia que ya tuvieron en el pasado.

El Nacional, eludiendo calificarlo de cine o teatro puesto que desempeñó las dos actividades, sigue siendo uno de los edificios más señeros de la ciudad, y un soberbio ejemplo de cómo se entendía una construcción monumental, generando un nuevo elemento del patrimonio de la ciudad de Melilla. 